\title{
ON THE CORPUSCULAR EMISSION THEORY OF STELLAR EVOLUTION
}

\author{
V. G. FESSENKOV and G. M. IDLIS \\ Astrophysical Institut, Alma Ata, USSR
}

Considerations regarding the evolutionary path of the main sequence stars depend essentially on the theorem of Vogt-Russell. According to this theorem the structure of stars with thermonuclear sources of energy $[\mathrm{I}-3]$ is determined uniquely by their masses and chemical composition, as characterised by the mean molecular weight

$$
\begin{gathered}
\mu=\frac{I}{2 \mathrm{X}+3 / 4 \mathrm{Y}+\mathrm{I} / 2 \mathrm{Z}} \\
(\mathrm{X}+\mathrm{Y}+\mathrm{Z}=\mathrm{I})
\end{gathered}
$$

$\mathrm{X}, \mathrm{Y}, \mathrm{Z}$ being the relative amounts of hydrogen, helium and of the mixture of the heavy elements [4].

It is well known that the theory of the internal structure of the stars and the mechanism of energy production yield two general relations between $\mathrm{L}, \mathfrak{N}, \mathrm{R}$ and $\mu$ (luminosity, mass, radius and mean molecular weight), whence eliminating $R$, we obtain a single relation

$$
\mathrm{L}=\mathrm{L}(\mathfrak{\Re}, \mathrm{X}) \text {, }
$$

(the admixture of heavy elements may be neglected [5]). [6]

At the same time observations show that along the first part of the main sequence the relation

$$
\mathrm{L} / \mathrm{L}_{\odot}=\left(\mathfrak{N} / \mathfrak{N}_{\odot}\right)^{4},
$$

(mass-luminosity relation) is satisfied sufficiently closely [7]. The decrease in the mass is apparently [8] accompanied by a decrease in $\mathrm{X}$ and a corresponding increase in the mean molecular weight [9].

$$
\mu=\mu(\mathfrak{N})
$$

This is in agreement with the hypothesis of stellar evolution along the main sequence by corpuscular emission $[6$, Io].

The rate of mass loss resulting from such corpuscular emission is approximately proportional to the luminosity of the star under consideration [II]

$$
\mathrm{d} \Im \mathrm{r} / \mathrm{dt}=-\mathrm{KL},
$$

but it exceeds the ordinary loss of mass by radiative emission nearly rooo times [I2, I3].

We have the following numerical estimate

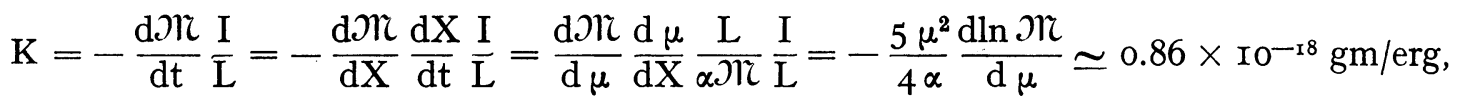

where $\alpha=6.4 \times$ Io $^{18} \mathrm{erg} / \mathrm{gm}$ is the energy generated by the transformation of one gram of hydrogen into helium [I4]. 
It is to be noted that the very existence of a statistical but exceedingly exact massluminosity relation for stars of the early types (which are of relatively very recent origin with rapidly changing brightness) testifies to the evolution of these stars along the upper part of the main sequence with a corresponding decrease in mass [6].

The decrease in the masses of the stars belonging to the upper part of the main sequence is accompanied by a corresponding retardation of rotation [I5-I7, II].

Moreover, it is to be noted that the increase in velocity dispersion for the stellar population I from type $\mathrm{OB}$ to type $\mathrm{F}$ indicates a corresponding systematic trend of the age characteristics [I8-25].

From the mass-luminosity relation (3) and the formula for corpuscular emission (5) one can easily determine the corresponding age of the stars under consideration.

$$
\tau=\frac{\mathfrak{M}_{\odot}}{3 \mathrm{KL} \odot}\left[\left(\frac{\mathfrak{M}_{\odot}}{\mathfrak{M}}\right)^{3}-\left(\frac{\mathfrak{M}_{\odot}}{\mathfrak{M}_{0}}\right)^{3}\right]
$$

where the value of the initial mass is important only for very young stars for which $\mathfrak{M} \simeq \mathfrak{M}_{\mathbf{0}}$.

During the period of existence of our Galaxy,

$$
\max \tau \simeq 5 \times 10^{9} \text { years }[26-28,3],
$$

the initially massive stars can diminish in mass down to

$$
\min \mathfrak{K}=\left(\frac{\mathfrak{K}_{\odot}}{3 \mathrm{KL}_{\odot} \max \tau}\right)^{\frac{1}{3}} \mathfrak{K}_{\odot} \simeq \mathrm{I}, \mathrm{I} \mathfrak{K}_{\odot}
$$

Hence evolution by corpuscular emission can be characteristic only of the stars of the first part of the main sequence. According to spectroscopic data $[29,30]$, our sun belongs to the second part of the main sequence for the evolution of which corpuscular emission is of no significance [3I].

Nevertheless, our sun continually emits corpuscular radiation, which profoundly affects the Earth, causing the aurorae and other phenomena. This solar corpuscular radiation has also a marked influence on comets and is apparently responsible for the almost complete absence of a lunar atmosphere.

These streams of corpuscles, however, cannot be detected photometrically at sufficiently great distances from the sun. Indeed, even the coronal rays, which are visible to distances of several solar radii can hardly be associated with such streams. Moreover, it is to be noted that all the photometric and polarizing properties of the Zodiacal light can be fully accounted for without recourse to an electronic component, although the interplanetary gas is almost fully ionized (90-95\%).

Nevertheless, the rôle of the corpuscular radiation of the sun cannot be neglected. Even the ordinary thermal dissipation of gases from a stationary corona with a possible temperature of $1.5 \times 10^{6}$ degrees leads to a total loss of $10^{17} \mathrm{gm}$ per years. Young, hot, rapidly rotating stars with their strong magnetic fields and their atmospheres almost in equilibrium under the force of gravity must undergo during their existence a relatively far greater loss of mass.

The stars of later types do not form any age sequence $[18,32]$ and do not show any systematic variation in chemical composition $[8,5]$. These stars are separated from the earlier types on the HR diagram at type $F$ and conserve their mass during evolution [3]. They do not reveal the rapid rotation or the strong magnetic fields with which intense corpuscular radiation is rightly associated [2I, 33]. 
It is to be noted [34] that some of the early type stars also rotate relatively slowly as, for instance, the bright stars in Pleiades and Hyades [35, 36]. It is possible that in the Pleiades [37] and perhaps in other clusters the axes of rotation have some preferential orientation and that the deficiency in rotational momenta is compensated by the general orbital revolution of the stars in these clusters [38]. The evolution of these stars with constant mass and increasing luminosity can account for the characteristic turn of the left end of the HR diagram, plotted for the open clusters, to the right and upwards from the general main sequence in the vicinity of the sun [3].

It can be assumed that the typical stars belonging to the first part of the main sequence originated at a uniform rate throughout the existence of our Galaxy and develop by way of corpuscular emission.

Hence, from the contemporary frequency function of absolute bolometric luminosity $[39,40]$ it is possible to deduce the initial luminosity function for new-born stars [14] :

$$
\psi(\mathfrak{N})=65 \times \mathrm{IO}^{-0,3 \mathrm{M}} \varphi(\mathfrak{\jmath})\left[\frac{\mathrm{d} \lg \varphi}{\mathrm{d} \mathfrak{N}}-0,3\right] .
$$

This function is in contradiction to earlier results [4I, 42], as it has two humps. The great majority of new-born stars enter the first part of the main sequence in the interval

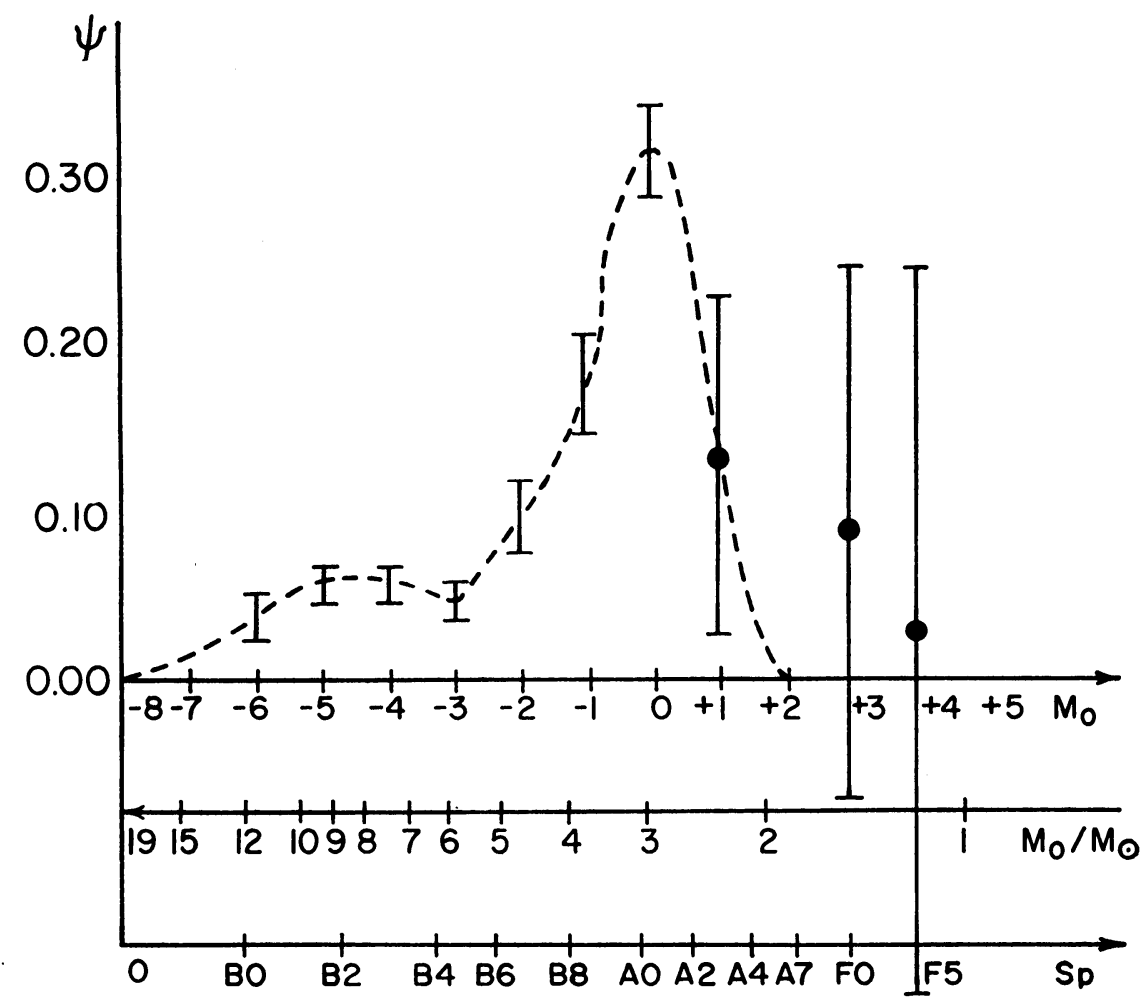

FIGURE I. The theoretical initial luminosity function for new-born stars, as a function of mass and luminosity, for early-type stars.

of absolute bolometric magnitudes $\mathrm{M}_{0}$ from -3 to $+\mathrm{I} .5$ (spectral types $\mathrm{B}_{4}+\mathrm{A}_{4}$, mass 6 to $\left.2 \mathfrak{M}_{\odot}\right)$. This fits in very well with the theoretical conclusion [43, 2I, 33, 44] regarding the 
existence of an upper limit of the mass in the process of stellar formation and evolution given by the formula :

$$
\mathfrak{k}_{\mathrm{K}}=25 \mathfrak{M}_{\odot} / \mu^{2} .
$$

As can be seen, a star passing through the stage of molecular hydrogen $(\mu=2)$ may have an initial mass no greater than $6 \mathfrak{N}_{\odot}$. The more massive stars must develop without passing through this stage, by the transformation of dwarfs into giants [21, 22, 33, 45] The corresponding process of accretion of interstellar matter should take place only in the depths of the maternal nebula where the star under consideration has a very small relative velocity. Accretion from without is impossible since the corresponding velocity is far too great, exceeding the parabolic value [46].

The most direct data on the initial luminosity function can be obtained from the study of associations of very young stars $[47,48]$. Table I presents a comparison of the observed luminosity functions $\varphi$ for the stellar associations in Cygnus [47] and Orion [49] with the mean statistical initial luminosity function $\psi$.

The agreement is very satisfactory. In the Cygnus association the secondary process of giant formation from dwarf stars is not evident, perhaps because of the absence of the necessary diffuse medium. In the Orion association, on the contrary, this process has produced a clearly visible secondary maximum in the same part of the luminosity function as in the initial theoretically computed function $\psi$.

Table I

The Predicted Initial Luminosity Function, and Observed Values

\begin{tabular}{|c|c|c|c|c|c|c|c|c|c|c|c|}
\hline$M$ & -6 & -5 & -4 & -3 & -2 & -1 & 0 & +1 & +2 & +3 & +4 \\
\hline$\psi$ & 0.04 & 0.06 & 0.06 & 0.05 & 0.10 & 0.18 & 0.32 & 0.13 & 0.0 & 0.0 & 0 \\
$\phi$ Cygni & 0.00 & 0.00 & 0.00 & 0.10 & 0.12 & 0.12 & 0.25 & 0.13 & 0.10 & 0.16 & 0 \\
$\varphi$ Orionis & 0.02 & 0.10 & 0.09 & 0.05 & 0.03 & 0.35 & 0.17 & 0.08 & 0.01 & 0.00 & 0 \\
\hline
\end{tabular}

The expansion of O-associations [50-59], consisting chiefly of stars of young spectral types can be also explained by corpuscular emission with a subsequent weakening of gravitational forces [6o].

It is to be noted that the gravitational perturbations due to the differentially rotating galactic medium with fluctuations of density lead to the following dispersion of peculiar velocities of the stars in the plane of the Galaxy [19] :

$$
\mathrm{V}(\bar{\tau})=\mathrm{V}_{\mathbf{0}}\left(\mathrm{I}+\bar{\tau} / \mathrm{t}_{\mathrm{E}}\right)^{\frac{1}{3}} .
$$

Here $V_{0}=I 0 \mathrm{~km} / \mathrm{sec}$ is the initial dispersion of the velocities of the youngest stars [6I], and $t_{E}$ is the corresponding relaxation time.

The conception of evolution by corpuscular emission allows us to calculate the average age $\bar{\tau}$ for each spectral group [14]. Table 2 illustrates the very good agreement of the observed dispersions of the peculiar velocities [32] with theoretical computations for the value of $t_{E}$ equal to $3.2 \times 10^{8}$ years. This value of $t_{E}$ corresponds to masses of the effective galactic inhomogeneities $\left(10^{5}\right.$ to $\left.10^{6}\right) \Re_{\odot}$ which are nearly the same as those of globular clusters, [62-64] of 
stellar clouds causing turbulence of the interstellar medium [2I, 33], of complexes of interstellar gaseous clouds [65] and of the Orion system $[49,66,67]$.

Table 2

The Age and Velocity Dispersion of the Stars

\begin{tabular}{|c|c|c|c|c|}
\hline Spectrum & $\mathrm{Oe}_{5}-\mathrm{B}_{5}$ & $\mathrm{~A}_{5}-\mathrm{A}_{9}$ & $\mathrm{Fo}_{0}-\mathrm{F}_{4}$ & $\mathrm{~F}_{5}-\mathrm{F}_{7}$ \\
\hline $\begin{array}{l}\quad \mathrm{M} \\
\bar{\tau} \text { years } \\
\mathrm{V} \mathrm{km} / \mathrm{sec} \text {, theory } \\
\mathrm{V} \mathrm{km/sec,} \mathrm{observed}\end{array}$ & $\begin{array}{c}<-2.6 \\
4 \times 10^{7} \\
10 \\
10\end{array}$ & $\begin{array}{c}1.6 \div 2.8 \\
1.0 \dot{\dot{x}} 10^{9} \\
16 \\
17 \pm 1\end{array}$ & $\begin{array}{l}2.8 \div 3.9 \\
2.5 \times 10^{9} \\
20 \\
20 \pm 1\end{array}$ & $\begin{array}{l}3.9 \div 4.35 \\
4.2 \times 10^{9} \\
24 \\
24 \pm \mathrm{r}\end{array}$ \\
\hline
\end{tabular}

In conclusion we enumerate the qualitative and quantitative arguments in favour of the theory of corpuscular evolution of the stars on the first part of the main sequence.

I) The systematic trend of the hydrogen and helium content; 2) the validity of the mass-luminosity relation notwithstanding the continued process of star formation; 3) the systematic trend of the velocities of rotation; 4) the systematic trend of the dispersion of peculiar velocities; 5) the peculiar shape of the general luminosity function; 6) the character of the luminosity function for stellar associations; 7) the expansion of O-associations; 8) the very simple relation between the corpuscular emission and the corresponding luminosity of the stars.

It is to be pointed out that all these arguments are only indirect ones and not all of them have the necessary precision. Nevertheless, direct arguments against the corpuscular theory of stellar evolution apparently do not exist at all.

The absence of effective mechanisms of sufficiently intense corpuscular emission is not an argument against our theory, inasmuch as many facts concerning the nonstationary state of different stars are as yet unexplained [68-70]. The absence of regular spherical envelopes of corpuscular luminous hydrogen around different hot stars is merely due to the circumstance that the emission from the sun and the stars is not homogenous, originating from isolated parts of their surfaces [7I].

R E F E R E N C ES

[I] C. F. von Weizsäcker, Phys. Zs., 39, 633, 1938.

[2] H. Bethe, Phys. Rev., 55, 434, I939.

[3] Les Processus Nucléaires dans les Astres, Bruxelles, I954.

[4] S. Chandrasekhar, An Introduction to the Study of Stellar Structure, 1939.

[5] D. A. Frank-Kamenetski, A. J. USSR, 31, 327, 1954 .

[6] V. G. Fessenkov. Report at the VIII Assembly of the I. A. U., Moscow, 1952. Publ. House, USSR Acad. of Sci.

[7] P. P. Parenago \& A. G. Massevitch, Sternberg Inst. Publ., 20, 8I, I95I.

[8] A. G. Massevitch \& P. P. Parenago, Sternb. Inst. Publ., 20, I30, I95 I.

[9] A. B. Severny, A. J. USSR, 31, 362, I954.

[10] A. G. Massevitch, Processus Nucléaires dans les Astres, Bruxelles, 1954, p. 170.

[II] V. G. Fessenkov, A. J. USSR, 26, 67, 1949.

[12] A. G. Massevitch, A. J. USSR, 26, 207, 1949.

[13] A. G. Massevitch, A. J. USSR, 28, 36, I95I.

[14] G. M. Idlis, A. J. USSR, 34, 755, I957.

[15] V. A. Krat, Comptes Rendus de l'Acad. d. Sciences de l'URSS, 59, 455, I948; Pulkovo Bulletin, 18, I, 1950.

[r6] D. J. Martynov, C. R. de l'Acad. d. Sciences de l'URSS, 60, I 149 , 1948. 
[I7] V. S. Safronov, A. J. USSR, 28, 244, I95I.

[18] L. Spitzer, Jr. and M. Schwarzschild, Ap. J., 114, 385, I95I.

[19] L. Spitzer, Jr., and M. Schwarzschild, Ap. J., 118, 106, 1953.

[20] D. E. Osterbrock, Ap. J., 116, 164, 1952.

[2I] A. I. Lebedinsky, Trans. and Conference on Cosmogony, 120, Moscow 1953.

[22] L. E. Gurevitch, Trans. 2nd Conference on Cosmogony, Moscow, 1953.

[23] L. E. Gurevitch, Problems of Cosmogony (Moscow), 2, I50, I954•

[24] T. A. Agekian, Memoirs of the Leningrad University, No. I53, math. series, No. 25, 48, 1952.

[25] G. M. Idlis, C. R. Acad. d. Sciences de l'URSS, 100, 635, 1955.

[26] R. Bouigue, C. R. Acad. Sci., 239, 866, I954.

[27] E. J. Opik, British Journ. Philosoph. Sci., 5, 203, I954.

[28] V. I. Baranov, 3rd Session of Commission for the Determination of Absolute Age of Geologic Formations, Supplement, 25I, Moscow 1955.

[29] E. K. Nikonova, Publ. Crimean Astrophysical obs., 12, 56, 1954.

[30] P. P. Parenago, Course of Stellar Astronomy, 3rd ed., Moscow, 1954.

[3I] S. K. Vsekhsviatsky, G. M. Nikolsky, E. A. Ponomarev, V. I. Cherednichenko, A. J. USSR, 32, 165, 1955.

[32] J. E. Ejnasto, Publ. Tartu. Observatory, 32, 371, 1954.

[33] A. I. Lebedinsky, Problems of Cosmogony, 2, 5, 1954, Moscow.

[34] C. W. Allen, Astrophysical Quantities, London, 1955.

[35] O. J. Eggen, Ap. J., 111, 65, 1950.

[36] O. J. Eggen, Ap. J., 111, 81, r950.

[37] E. van Dien, Journ. Royal Astr. Soc., Canada, 42, 249, 1948.

[38] P. P. Parenago. Studies in Stellar Astronomy in the USSR. Moscow, 1955. Publishing House of the USSR Academy of Sciences, p. 5.

[39] E. E. Salpeter, Ap. J., 121, I6I, I955.

[40] A. G. Massevitch, A. J. USSR, 33, 216, 1956.

[4I] P. P. Parenago, A. J., 28, 93, I95I.

[42] S. A. Kaplan, A. J. USSR, 30, 39I, I 953.

[43] A. I. Lebedinsky, C. R. Acad. Sci. de l'URSS, 79, 415, 195 I.

[44] G. M. Idlis, Cosmical Matter, Moscow, 1957.

[45] L. E. Gurevitch, Problems of Cosmogony, 3, 94, 1954.

[46] G. M. Idlis, Bulletin of Astroph. Inst. Acad. Sci. Kazakh. SSR, 2, 34, 1956.

[47] V. A. Ambartsumian, Transactions of the and Conference on Cosmogony 9, 1953, Moscow.

[48] V. A. Ambartsumian, Discours Introductif au Symposium sur l'évolution des étoiles. VIII Assemblée de l'U. A. I. Rome, 1952.

[49] P. P. Parenago, Sternb. Inst. Publ., 25, 3, 1954.

[50] V. A. Ambartsumian, A. J. USSR, 26, 3, 1949.

[5I] A. Blaauw, B. A. N., no 433, 1952.

[52] J. H. Oort, M. N. Astr. Soc. of South Africa, 11, 91, 1952.

[53] B. E. Markarian, Burakan Communications, No II, 3, 1953.

[54] B. E. Markarian, Burakan Communications, No II, I9, I 953.

[55] I. M. Kopylov, C. R. Acad. Sci. l'URSS, 90, 975, 1953.

[56] A. Blaauw, W. W. Morgan, Ap. J., 117, 256, 1953.

[57] H. F. Weaver, P. A. S. P., 65, 132, 1953.

[58] A. F. Torondjadze, Abastumani Bulletin, No I 5, I $15,1953$.

[59] K. A. Steins, M. K. Abele, A. J. USSR, 35, 82, 1958.

[6o] G. M. Idlis, Bull. Astroph. Inst., Acad. Sci. Kazakh. SSR, 2, 4I, 1956.

[6I] H. Nordström, Lund Obs. Medd. Ser. II, 79, I6I, I936.

[62] N. N. Parijsky, Russian Astr. Journ., 3, 10, 1926.

[63] O. Hachenberg, Zs. f. Ap., 18, 49, 1939.

[64] H. M. Johnson, Ap. J., 115, I 24, 1952.

[65] G. A. Shajn, C. R. Acad. Sci. de l'URSS, 93, 993, I953.

[66] G. M. Idlis, Bull. Astroph. Inst. Acad. Sci. Kazakh SSR, 4, 3, I957.

[67] T. K. Menon, A. J., 62, 27, I957.

[68] Transactions of the 4th Conference on cosmogony, Moscow, 1955. Publ. House USSR Acad. of Sciences.

[69] V. A. Ambartsumian. (Communications for the Symposium on Non-Stable Stars), Moscow, I955.

[70] Non-stable stars. Erevan, 1957 .

[71] Physics of Solar Corpuscular Streams and Their Influence on the Upper Atmosphere of the Earth, Moscow 1957. 


\section{Discussion}

Fessenkov : In response to a question on who deduced, and how, the formula (II) for the upper limit of stellar mass, the formula was deduced by the first time by Lebedinsky on thermodynamical grounds. It is given in C.R. Acad. Sci. URSS, 79, 4I5, I95I, and in Problems of Cosmogony, 2, I954, Moscow. Hovewer the formula was derived in a qualitative way by G. M. Idlis in his book Cosmical Matter (in Russian), I957, Moscow.

Schatzman : I thought, several years ago, that there was no difficulty in accepting the suggestion that mainsequence stars were losing mass at a high rate. I have studied in I95I free waves in an atmosphere. For a certain range of wave lengths, free waves in a stellar atmosphere are unstable and can certainly be at the origin of some corpuscular losses. However, that process seems very inefficient, and, in case of the sun, can at most supply the amount of matter which is lost by the corona.

I can imagine loss of mass due to rotation, during the last stages of the contraction of a star, the loss of mass which occurs just after this one, as suggested by Krat, and due to the inertia of the radiation of the star; I can imagine the physical process of a continuous loss of mass by giants and supergiants as a consequence of the shock waves running through the atmosphere and chromosphere of these stars. But I must confess that I do not see how what would be the physical process leading to the mass loss which has been assumed, for the first part of the main sequence. 